\title{
Untersuchungen zur Schaffung neuartiger Sensor-Aktor- Lösungen für die Bauwerkssanierung
}

\author{
F. Gerlach, K. Ahlborn, W. Vonau \\ Kurt-Schwabe-Institut für Mess- und Sensortechnik e.V. Meinsberg \\ Kurt-Schwabe-Straße 4, D-04736 Waldheim, www.ksi-meinsberg.de, \\ gerlach@ksi-meinsberg.de
}

\begin{abstract}
:
Elektrodensysteme werden auf vielfältige Art und Weise für elektrophysikalische Entfeuchtungs- und Entsalzungsverfahren in der Bauwerkssanierung verwendet. Auf Grund der hohen elektrochemischen Belastung weisen kommerzielle Elektroden ein teilweise instabiles Verhalten auf. Damit kommt es zu Veränderungen der methodischen Randbedingungen und zu Problemen bei der Definition von objektspezifischen Abbruchkriterien von Sanierungsprozessen. Um dies zu vermeiden, bedarf es einer kontinuierlichen Charakterisierung des Baukörpers und des Zustandes der darin eingebrachten Elektroden. Der vorliegende Beitrag stellt für die Bauwirtschaft geeignete Sensor-Aktor-Systeme und Methodiken zur Feuchtebestimmung in der Bausanierung vor.
\end{abstract}

Key words: Sensor-Aktor-System, Bauwerkssensorik, Entfeuchtung, Entsalzung.

\section{Einleitung}

Sowohl bei Neu- als auch bei Altbaubeständen kann eindringende Feuchte nicht nur zu nachhaltigen Gebäudeschäden, sondern durch nachfolgende Schimmelbildung auch zu gesundheitlichen Beeinträchtigungen führen, deren Folge zeit- und kostenintensive Sanierungsmaßnahmen sind. Der Innovationsgehalt von zu entwickelnden Entfeuchtungs- und Entsalzungsmethoden [1- 6] wird wesentlich durch die Sensorintegration geprägt. Dies setzt voraus, dass die automatisierte Analyse des jeweils aktuellen Mauerwerkszustandes wesentlich qualifizierter durchgeführt wird, als das bisher geschieht. Die Kombination der etablierten Verfahren mit modernen elektrochemischen Untersuchungsmethoden ermöglicht zusätzlich die Erfassung und Bewertung der Elektrodenzustände. Insgesamt soll die neuartige Sensor-Aktor-Methode [7] und die Elektrodenkombination einen automatisierten Betrieb von Entfeuchtungs- und Entsalzungsanlagen bei gleichzeitiger Verlängerung der Lebensdauer der Elektroden ermöglichen.

\section{Lösungsweg}

Die Charakterisierung potentieller Arbeitselektroden wurde unter Laborbedingungen hinsichtlich Korrosionsverhalten, Langzeitstabilität und Möglichkeiten einer optimierten Mauerankopplung durchgeführt.

Da die physikalischen Verfahren in der Regel auf einem aktiv induzierten Stromfluss zwischen mindestens zwei Elektroden basieren, führt dies in Verbindung mit den vorhandenen Salzfrachten im Mauerwerk zu einer erheblichen Korrosionsgefahr der Arbeitselektroden. Kommerziell werden derzeitig sowohl metallische als auch polymergebundene Elektroden verwendet. Im vorliegenden Projekt wurden darüber hinaus kohlenstoff- und titanbasierte Elektroden, platinbeschichtete Keramikelektroden, Elektroden aus korrosionsfesten Edelstahl (1.4571) und beschichtete Elektroden auf der Grundlage von Titan charakterisiert. Basis des Messaufbaus ist eine Dreielektrodenanordnung, angelehnt an eine klassische Korrosionsmesszelle (Fig. 1).

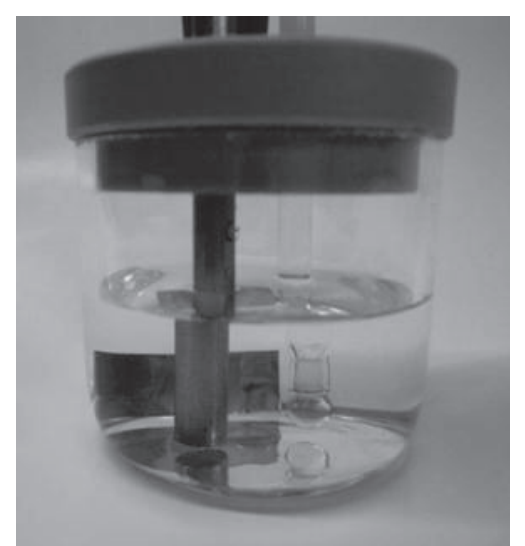

Fig. 1. Messaufbau zum Materialscreening als 3Elektroden-Messzelle (Elektrolyt 0,1 $\mathrm{M} \mathrm{KCl}$, $U p=10 \mathrm{mV} \sim$ ) 
Lediglich das Messvolumen wurde verringert und an die Elektrodengeometrie angepasst. Für die Charakterisierung wurde eine 3-ElektrodenMesszelle mit einer Platin-Gegenelektrode und einer $\mathrm{Ag} / \mathrm{AgCl}, \mathrm{Cl}_{\text {ges. }}^{-}$-Bezugselektrode verwendet. Die Bestimmung des Ruhepotenzials und des Impedanzspektrums erfolgte in einer $0,1 \mathrm{M} \mathrm{KCl}$ Lösung (Fig. 2). rungsform und Einbrandbedingungen um über eine Dekade.

Ein weiterer zu berücksichtigender Aspekt ist die Ankopplung der Arbeitselektroden im Mauerwerk. Es wurden Laborversuche an einem durchfeuchteten Vollziegel durchgeführt (Fig. 3), bei dem identische graphitbeschichtete Edelstahlelektroden mit unterschiedlichen Materialen eingebracht wurden. Neben kommerziell verfügbaren Putz- und Maurermörtel kamen mit Graphit

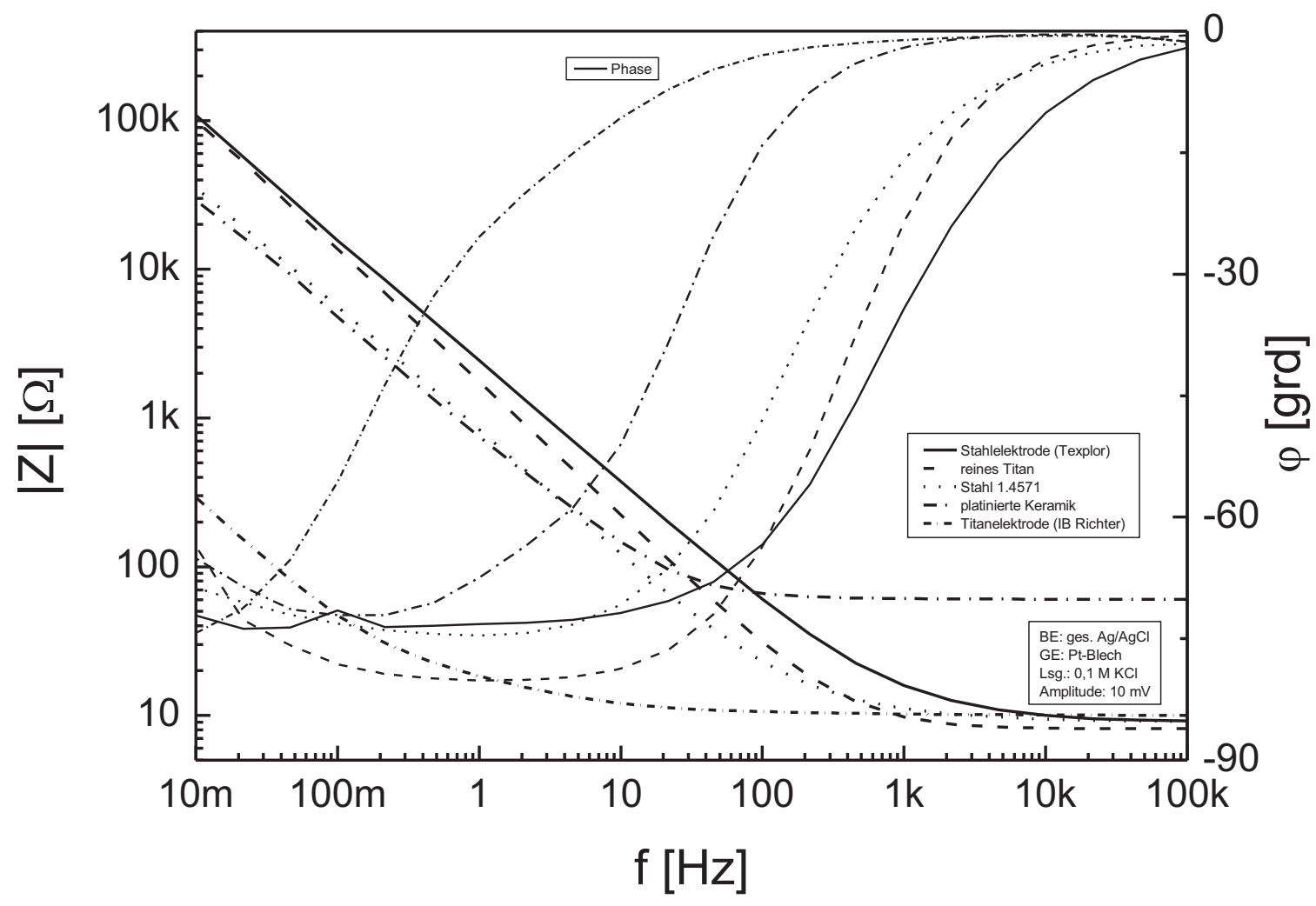

Fig. 2. Elektrodencharakterisierung mittels Aufnahme von Impedanzspektren

Sowohl der platinierte Keramikstab als auch die kommerzielle Titanelektrode zeigen sehr stabile Ruhepotenziale und die niedrigsten Polarisationswiderstände. Durch die bei diesen Elektroden verwendeten Werkstoffkombinationen besitzen die Arbeitselektroden auch im polarisierten Zustand eine hohe Korrosionsbeständigkeit. Da diese Elektroden in der Herstellung sehr aufwendig sind, wurde alternativ versucht, den Polarisationswiderstand der korrosionsstabilen Arbeitselektroden auf der Grundlage von Titanlegierungen mittels Oberflächenbeschichtungen zu senken. Die konfektionierten Titanelektroden erfuhren eine Oberflächenmodifikation auf der Basis von Graphitlack. Diese Maßnahme senkt den Polarisationswiderstand gegenüber der metallischen Grundelektrode je nach Ausfüh-

und Salz modifizierte Gipse sowie Dünnbettmörtel mit einem hohen Wasserbindevermögen zum Einsatz. Alle eingesetzten Systeme zeigten im Gegensatz zu der Variante ohne Zusatzmaterial ausreichend niedrige Übergangswiderstände bei unterschiedlichen Trocknungszuständen. Die salzbeladenen Systeme neigen während des Trocknungsvorganges zur Salzauskristallisation und zur Segregation unterschiedlicher Salzfraktionen und schieden deshalb aus. Von allen Systemen ließ sich der Dünnbettmörtel auf Grund der guten Verarbeitungseigenschaften und der feinen Kornstruktur sehr gut mittels Kartuschenpistolen mit den Arbeitselektroden in den Ziegel einbringen. Dieses Verfahren wurde ebenfalls bei der Applikation von Elektroden in einem Modellmauerwerk und bei der Durchführung von Feldtests in einer Wohnanlage angewendet. 


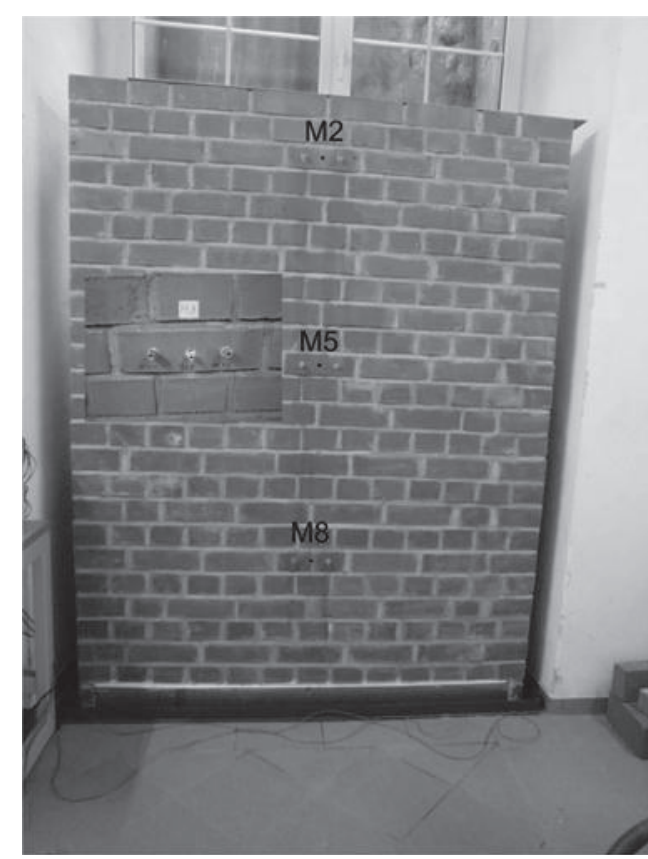

Fig. 3. Modellmauerwerk für Labortests mit Positionierung der Messpunkte M2, M5 und M8

Um applikationsnahe Bedingungen nachzustellen, wurde ein Vollziegelmauerwerk aus NF Mauerziegeln Mz 20/2 in Mörtelgruppe Ila mit einer Wanddicke von $36,5 \mathrm{~cm}$, einer Breite von $166 \mathrm{~cm}$ und einer Höhe von $215 \mathrm{~cm}$ im Kreuzverband erstellt (siehe Fig. 3).

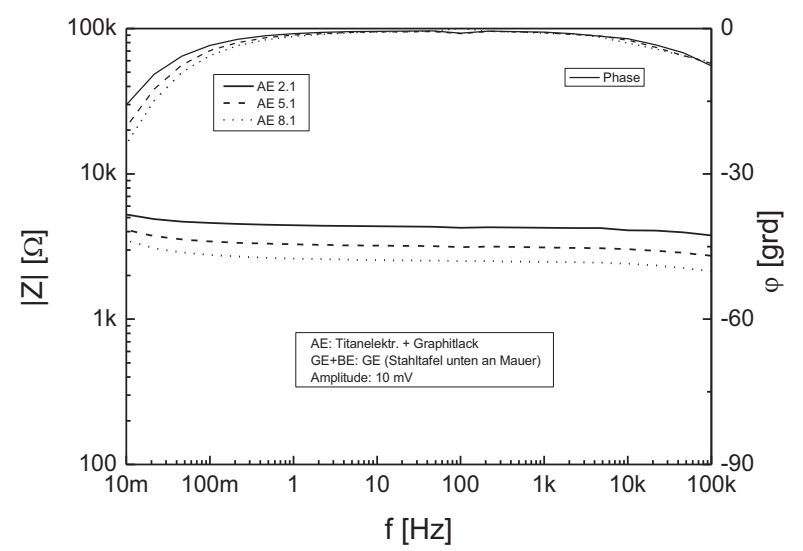

Fig. 4. Impedanzspektroskopie (trockene Mauer M2, M5 und M8)

Eine durchgehende Kunststoffwanne (unter der Mauer) ermöglicht in Verbindung mit einer Schlauchpumpe und einer Zeitsteuerung das kontrollierte Befeuchten der Mauer oder Teilen davon. Es sind in einem ersten Schritt in 3 unterschiedlichen Höhen Arbeits- und Bezugselektroden eingebracht worden, um über Potenzialbestimmungen und Impedanzmessungen eine umfassende Charakterisierung des Mauerwerkkörpers zu gewährleisten.
In Fig. 4 ist ein typischer trockener Mauerzustand in einem Impedanzspektrum dargestellt. Die Phasenlage ist über einem weiten Frequenzbereich nahe $0^{\circ}$, und verhält sich über lange Zeiträume sehr stabil. Die Modellmauer wurde verschiedenen Feuchtezuständen ausgesetzt und die Elektrodenreaktion ausgewertet. Figur 5 zeigt eine Darstellung verschiedener Feuchtezustände in Auswertung von Impedanzen bei $10 \mathrm{~Hz}$.

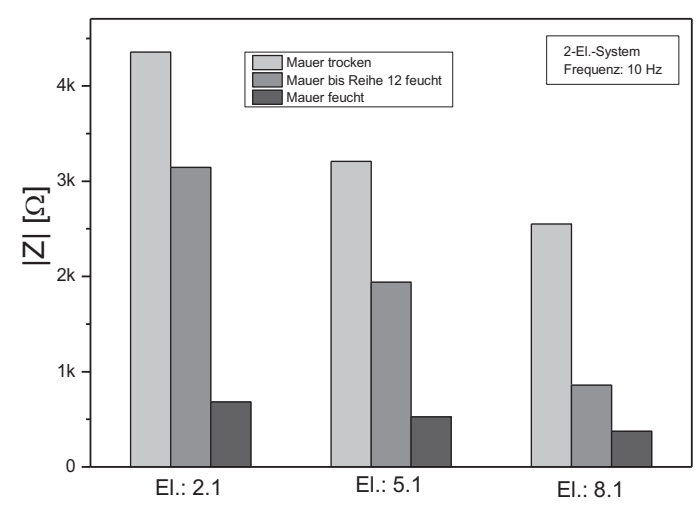

Fig. 5. Auswertung von Impedanzen bei unterschiedlichen Mauerzuständen

Eine direkte Proportionalität zwischen dem Feuchtegrad eines Baukörpers und impedimetrischen Messungen, wie sie im Rahmen dieses Projektes durchgeführt wurden, ist nicht nachweisbar, da die Temperatur und der Salzgehalt nachweislich einen wesentlichen Einfluss auf die Volumenleitfähigkeit von Baukörpern besitzen.

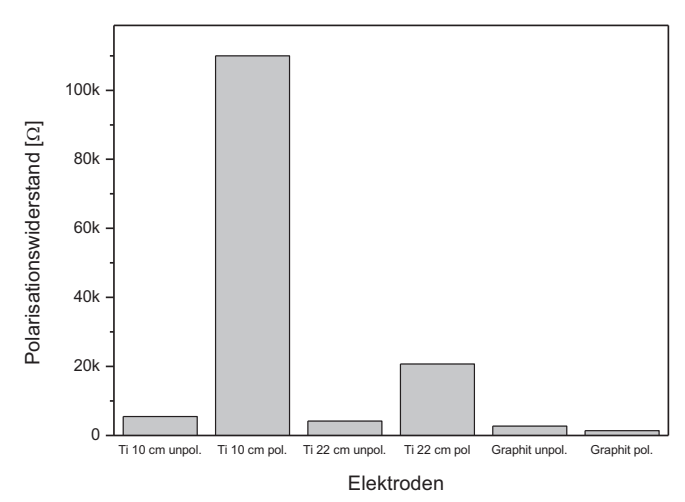

Fig. 6. Vergleich der Polarisationswiderstände in Abhängigkeit vom Elektrodenaufbau (Stromdichte) 
Dennoch konnten Korrelationen zwischen den impedimetrischen Messungen und den Referenzmessungen mit der Mikrowellenmethode [8] (MOIST 300 / Fa. HF Sensor $\mathrm{GmbH}$ ) gefunden werden, vorausgesetzt, es sind keine Lösevorgänge von Salzfrachten beteiligt.

Der Einsatz der Elektrodenmaterialien für bautypische Applikationen zur Charakterisierung und Sanierung von Baukörpern ist meist verbunden mit einer hohen elektrochemischen Belastung der Elektroden. Diese kann nur über eine Vergrößerung der Elektrodenfläche und somit einer Reduzierung der Stromdichte vermindert werden. Experimentelle Untersuchungen hierzu wurden mit unterschiedlichen zylindrischen Titanelektroden und flächigen Graphitelektroden durchgeführt (Fig. 6).

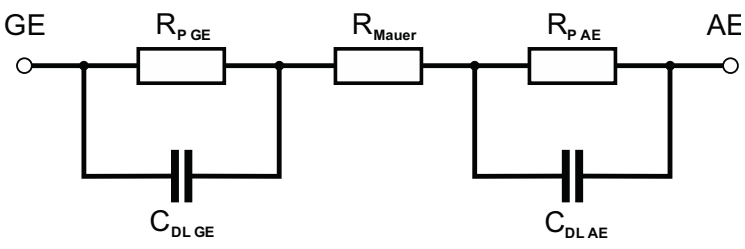

Fig. 7. Modellannahme von in einem Mauerwerk eingebrachte Arbeits- und Gegenelektroden
In der Impedanzspektroskopie ist es üblich, die komplexen Systeme mit Einzelkomponenten zu simulieren, um die physikalischen Eigenschaften mit guter Näherung abzubilden. Diese Eigenschaften können dann frequenzunabhängig den einzelnen Komponenten zugeordnet werden. Grundlage bildet eine Modellannahme, wie in Fig. 7 dargestellt.

Die wichtigsten Elemente sind die Mauer- und Polarisationswiderstände $\left(R_{\text {Mauer }}, R_{P}\right.$ GE und $\left.R_{P A E}\right)$ der Elektroden und deren sich ausbildende Doppelschichtkapazitäten $\left(\mathrm{C}_{\mathrm{DL}} \mathrm{GE}\right.$ und $\left.\mathrm{C}_{\mathrm{DL}} \mathrm{AE}\right)$. Mit diesem Ersatzschaltbild lassen sich die Bedingungen an der Modellmauer, wie in Fig. 8 dargestellt, mit hoher Genauigkeit nachbilden.

Die potenziodynamischen Untersuchungen wurden in einem nächsten Schritt mit polarisierten und unpolarisierten Elektroden am Modellmauerwerk durchgeführt. Dabei konnten folgende Werte den Ersatzschaltbildelementen zugeordnet werden: $\quad R_{\text {Mauer }}=2,87 \mathrm{k} \Omega, \quad R_{P G E}=1,64 \mathrm{k} \Omega$, $\mathrm{R}_{\mathrm{PAE}}=23,95 \mathrm{k} \Omega, \quad \mathrm{C}_{\mathrm{DL}} \mathrm{GE}=1,66 \mathrm{nF}$ und $\left.\mathrm{C}_{\mathrm{DL} A E}=266 \mu \mathrm{F}\right)$.

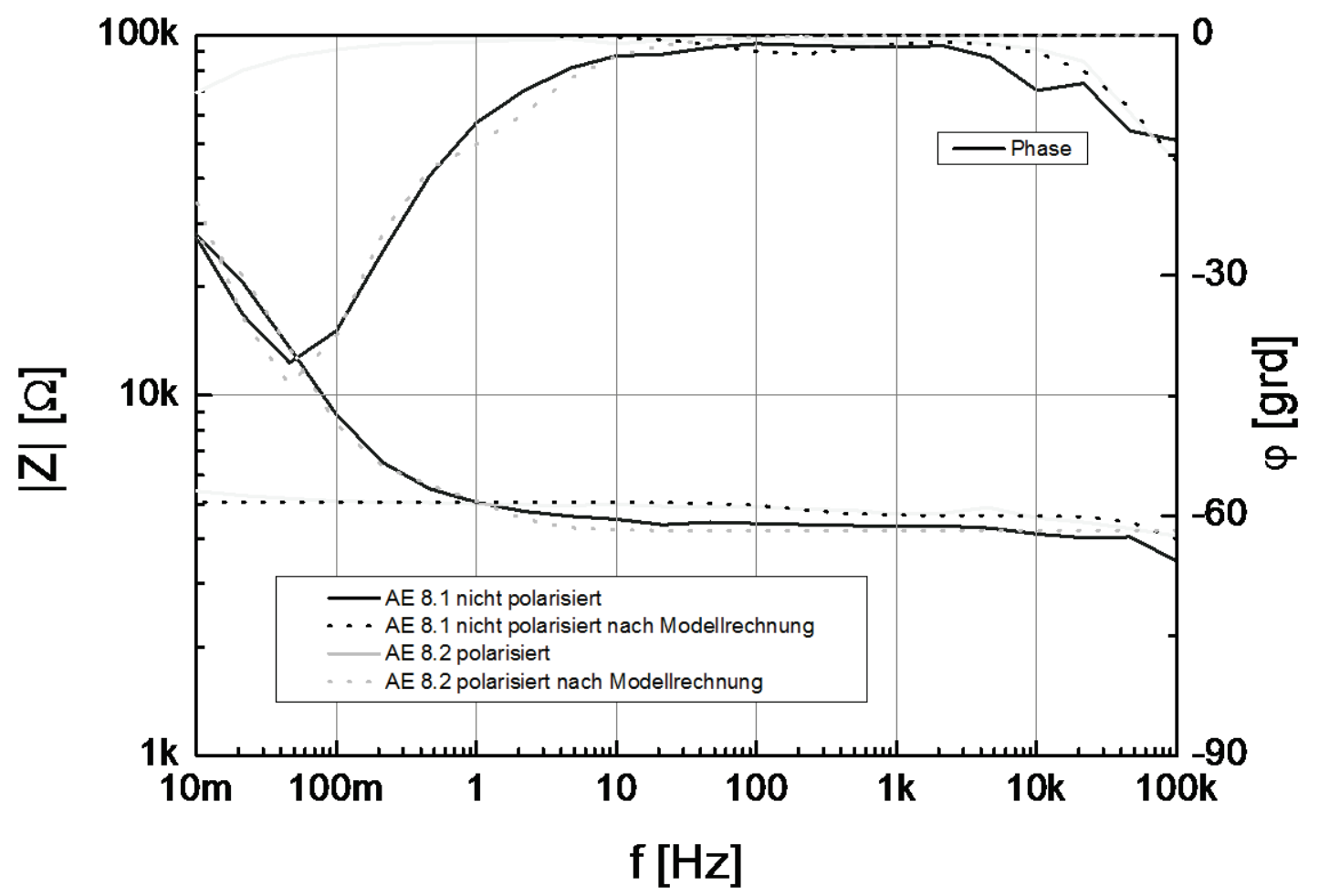

Fig. 8. Darstellung der Modellierung nach dem Ersatzschaltbild (Fig. 7) von polarisierten und unpolarisierten Elektroden 
Neben der Impedanzspektroskopie kamen die zyklische Voltammetrie und die Chronoamperometrie zur Charakterisierung der Elektroden am Modellmauerwerk zum Einsatz. Mit beiden Methoden kann man den Elektrodenzustand näher charakterisieren. Bei der zyklischen Voltammetrie ist man in der Lage, durch kleine Spannungsänderungen um das Ruhepotenzial den Polarisationswiderstand der Elektrode abzuschätzen. Problematisch bei dieser Methode ist der variable Einfluss von Verschiebungsladungsträgern durch unterschiedliche Scangeschwindigkeiten. Die Fig. 9 zeigt zyklische Voltammogramme polarisierter und unpolarisierter Elektroden mit verschiedenen ScanGeschwindigkeiten. Die Verhältnisse des Polarisationswiderstandes bleiben bei polarisierten und unpolarisierten Elektroden bei unterschiedlichen Scan-Geschwindigkeiten nahezu gleich.

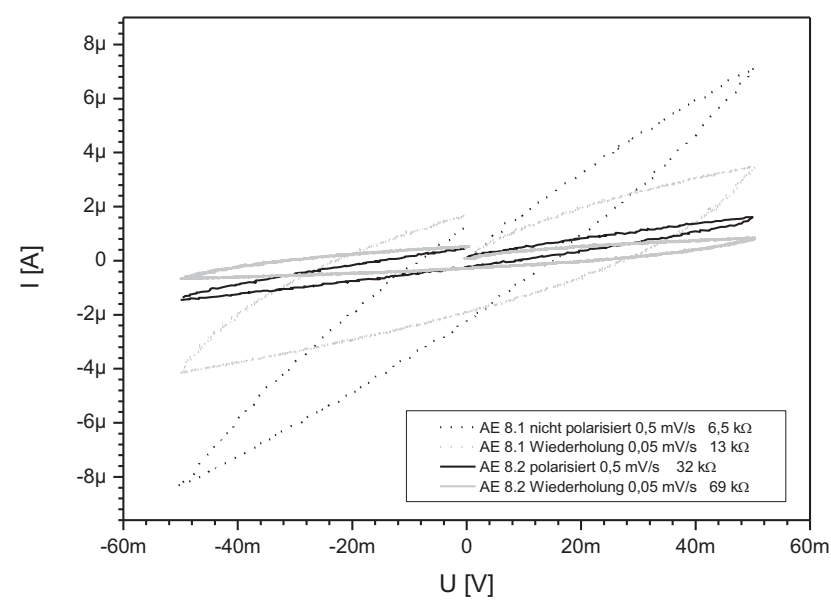

Fig. 9. Zyklische Voltammogramme nicht polarisierter ( $A E$ 8.1) und polarisierter Elektroden ( $A E$ 8.2) bei unterschiedlichen Scan-Geschwindigkeiten von $0,5 \mathrm{mV} / \mathrm{s}$ und $0,05 \mathrm{mV}$

Eine weitere potenziodynamische Methode ist die Chronoamperometrie. Hierbei wird, ebenfalls ausgehend vom Ruhepotenzial, eine relativ kleine positive und negative Gleichspannung auf das System aufgeprägt und aus der resultierenden Stromantwort der Polarisationswiderstand berechnet. Diese Methode ist in Fig. 10 dargestellt. Um reproduzierbare Ergebnisse zu erhalten, muss in Abhängigkeit vom zu untersuchenden Baukörper die optimale Messzeit ermittelt werden. Der Vergleich der unterschiedlichen elektrochemischen Methoden ergab eine gute Charakterisierungsmöglichkeit von polarisierten und unpolarisierten Arbeitselektroden. Für die Implementierung von Elektrodencharakterisierungsmethoden in ein aktives Steuergerät zur Entfeuchtung von Baukörpern bieten potenziodynamische Verfahren klare Vorteile gegenüber

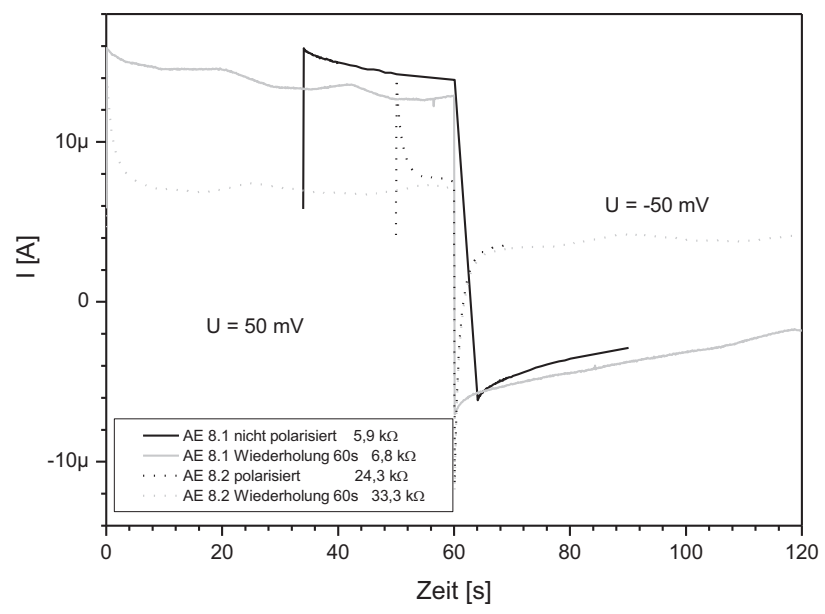

Fig. 10. Chronoamperometrische Untersuchung nicht polarisierter (AE 8.1) und polarisierter Elektroden (AE 8.2) am Modellmauerwerk (Potenzialsprünge +/- $50 \mathrm{mV}$ vom Ruhepotenzial)

der klassischen Impedanzspektroskopie, da hier der messtechnische Aufwand wesentlich geringer ist und die Messaufgabe meist mit den eingesetzten Einchipmikrorechnern und der geeigneten AD/DA-Wandlertechnik leichter realisierbar ist.

\section{Zusammenfassung}

Elektrochemische Charakterisierungsmethoden gestatten die Beurteilung des Baukörpers und der Sensor-Aktor-Komponenten. Diese Methodik konnte in einem 5-monatigem Feldtest zur Trocknung und Entfeuchtung von Kellern einer Wohnanlage in Berlin angewendet werden. Unter realen Praxisbedingungen zeigte sich die Anwendbarkeit von elektrochemischen Methoden und Verfahren zur Charakterisierung der Baukörper und der eingebrachten Sensor-Aktor-Elemente. Sowohl die Impedanzmessungen als auch die potenziodynamischen Methoden wie zyklische Voltammetrie und Chronoamperometrie lassen sich zur Charakterisierung von SensorAktor-Komponenten heranziehen und liefern vergleichbare Informationen. Damit stehen potentiellen Geräteentwicklern auf der Grundlage der Auswertung von Strom- und Spannungswerten ähnlich verwertbare Informationen zur Verfügung, ohne auf die gerätetechnisch aufwendige Auswertung im Zeitbereich in Form der Impedanzspektroskopie zurückgreifen zu müssen. Dies hat direkte Auswirkungen auf die Entwicklung von effektiven Methoden zur Entfeuchtung und Entsalzung von Baukörpern. 


\section{Literatur}

[1] K. Ahlborn, F. Berthold, W. Vonau, H. Grünzig, U. Schneck, H. Jahn, J. Köhler,

Modular corrosion measurement system (CMS) for electrochemical NDT, In: M. Grantham, V.

Mechtcherine, U. Schneck (Eds.): Concrete Solutions 2011. Proceedings of the $4^{\text {th }}$ International Conference on Concrete Repair. Dresden, 26.-28. September 2011. Boca Raton, CRC Press, 2011

[2] S. Herrmann, F. Berthold, W. Vonau, H. Grünzig, U. Schneck, Messmodule zur Chloridbestimmung bei der Stahlbetonsanierung, GVC/DECHEMA-Jahrestagungen 2005. Wiesbaden, 06.-08.09.2005, Kurzfassung des Beitrages in: Chemie Ingenieur Technik 2005, 77, No. 8, 1115, P 10.02, Poster und Postervortrag

[3] U. Schneck, H. Gruenzig, W. Vonau, S. Herrmann, F. Berthold, Chloride measuring unit for the improvement of security and performance of the electrochemical chloride extraction, Concrete Solutions Conference. Saint-Malo, France, 27.-29.06.2006, Vortrag

[4] W. Vonau, S. Herrmann, F. Berthold, Entwicklung eines Chloridmessmoduls (Teilthema), Schlussbericht zum Verbundprojekt "Prozessüberwachung beim elektrochemischen Chloridentzug aus Stahlbeton", SAB 6960/1092, 2004

[5] W. Vonau, S. Herrmann, F. Berthold, T. Bachmann, Corrosion processes in microsystems and sensor technology. In O. Fors', J. Aromaa, L. Selin: KSCS 2004, 4 th Kurt Schwabe Corrosion Symposium "Mechanismus of Corrosion Prevention", Helsinki University of Technology, Espoo, Finland 2004, Proceedings p. 266-274

[6] F. Berthold, K. Ahlborn, W. Vonau, Anwendung elektrochemischer Untersuchungsmethoden am Stahlbeton mittels spezieller Aufsatzzelle. tm Technisches Messen 79 (2012) 12, 531-541

[7] F. Gerlach, K. Ahlborn, W. Vonau, Electrodes for electro physical dehumidification of brick masonry 63rd Annual ISE Meeting, 19.-24.08.2012, Praque, Czech Republic

[8] J. Weber, V. Hafkesbrink (Hrsg.), Bauwerksabdichtung in der Altbausanierung, Vieweg+Teubner Verlag, GWV Fachverlage $\mathrm{GmbH}$, Wiesbaden, 2008

\section{Danksagung}

Das ZIM-Vorhaben „Entwicklung eines modular aufgebauten Kombigerätes zur Früherkennung von Mauerfeuchte und zur automatischen Steuerung des Entfeuchtungsprozesses“ (FKZ 2218308DF0) wurde über die AiF im Rahmen des Zentralen Innovationsprogramm Mittelstand (ZIM) vom Bundesministerium für Wirtschaft (BMWi) und Technologie aufgrund eines Beschlusses des Deutschen Bundestages gefördert. 